\title{
"Upright" orientations of forms change with subject age and with features of form
}

\author{
M. JOSEPH SCHALLER \\ University of Wisconsin-Madison, Madison, Wisconsin 59706 \\ and \\ LAUREN JAY HARRIS \\ Michigan State University, East Lansing, Michigan 48829
}

\begin{abstract}
This research investigated the role of certain intrinsic features of two-dimensional nonrepresentational forms in their judged "upright" orientation. The forms were individually projected by an apparatus that allowed rotation through the 360-deg range. The subjects (from kindergarten, 2nd, 4th, 6th, college grades) were asked to place each form in "upright" orientation. For some forms, these chosen orientations were the same for all age groups, apparently reflecting the universal and equal effect of certain intrinsic features. For other forms, chosen orientation changed systematically across age, in a manner possibly reflecting the influence of reading-scansion habits that changed with age. The intrinsic features related to upright judgments were symmetry, polar axes, texture lines, taper, and flat bottoms. Results partly disconfirm the hypothesis that forms are judged "upright" with their "focal portions" at the top.
\end{abstract}

It is now difficult to accept the early view (Koffka, 1924; Stern, 1930) that children are "insensitive" to the orientation of form; a variety of more recent studies have demonstrated that young children do respond to orientation, although not always in the same way as older children and adults. First, children, like adults, can detect differences in orientation of identical forms (e.g., Wohlwill \& Weiner, 1964). but, unlike adults, more often may have different criteria for, or notice but fail to comment on, differences in orientation of the same form (e.g., Davidson, 1934, 1935). In addition, when compared with adults, younger children's ability to remember or identify some types of forms (e.g., faces, cf. Brooks \& Goldstein, 1963; Goldstein \& Chance, 1964, 1965) seems to be disrupted more by an uncommon orientation, although the direction of this effect is uncertain (Goldstein, Note 1; Schaller, Note 2; Wright \& Schaller, Note 3). Finally, under some conditions, young children's judgments of orientation are more consistent than older children's

This report is based in part on a dissertation submitted in partial fulfillment of the requirements of the $\mathrm{PhD}$ degree at Michigan State University by the first author under the direction of the second author. Portions of this research were reported at the Biennial Meetings of the Society for Research in Child Development at Minneapolis, April 1971. Requests for reprints should be addressed to M. Joseph Schaller, Department of Psychology, University of Wisconsin, Charter at Johnson Streets, Madison, Wisconsin 53706. We thank Ellen Strommen, Charles Hanley, Paul Bakan, Merrill Mitler, Romie Schaller, and Principal Kent Thibideau and the teachers of Midway School in Holt, Michigan, for their help and cooperation.
[Ghent (Braine), 1961; Harris \& Schaller, 1971, Note 4, 1973].

These facts have implications for a variety of research questions in perceptual development, such as the investigation of objer $t$ constancy and shape and slant perception (cf. Harris \& Allen, 1974) or analyses of letter and word reversals in reading. They also have been seen as evidence of the nature of processing of visual information-in particular, as an indication that visual information is processed sequentially and that the directon of the sequences changes with age (Ghent, 1961; Braine, 1972; Schaller \& Dziadosz, Notes 5 and 6). In this regard, Braine (Ghent, 1961) has argued that the high agreement among 4- and 5 -year-olds in judged orientation of forms reflects a tendency to view the form as upside down when a "focal point" of the form is in the lower half. Young children, the argument continues, are relatively restricted in their choice of processing sequences so that their usual top-to-bottom processing is disrupted if their attention is drawn immediately to the bottom by the "focal portion of the form." Older children are somewhat more flexible and can begin to attend to the form in a number of ways. They, therefore, are less likely to experience disruption, and consequently do not agree so readily as younger children when the form is not upright (Braine, 1965).

We have demonstrated that children do strongly agree which way the forms Braine used are upside-down, confirming Braine's findings (Ghent, 1961). In addition, we have found that children agree even more strongly which way the forms are right-side-up (Harris \& Schaller, 1971, 1973). The 
current research addresses the question of explanation: do judgments of orientation represent a basic perceptual processing strategy. as Braine has argued, or can they be explained in some other way?

There are several other possibilities: perhaps children find instructions to place nonrealistic forms upright to be ambiguous, and faced with such ambiguity they simply orient the forms according to their personal liking for one orientation rather than another. At least for kindergartners, this possibility is unlikely. When asked to indicate which way they most "liked" the forms, rather than the way they would be upright, kindergartners responded too variably and too often included oblique orientations to account for the consistent orientation of upright orientation found previously (Harris \& Schaller, Note 4).

Specific experiences could affect orientation judgments too. Some of the forms Braine used resemble letters, leading to the possibility, as we have argued (Harris \& Schaller, 1971, 1973), that experience with the alphabet could underlie orientation judgments for these forms. For example, a substantial number of subjects in our previous study oriented open circles as though they were the letter "C.",

More general experiential hypotheses are possible. Associations between the forms and similar-shaped real-life objects may underlie orientation judgments. At least with college-age subjects, however, this possibility has not been supported. Harris and Gutkin (unpublished data) had subjects make associations to forms prior to judging their orientations. For most forms, the associations, while appropriate to the orientation in whch the form was presented, was irrelevant to the subjects' subsequent decision as to whether the form in that orientation was right-side-up or not.

A second "experiential" explanation is also possible: perceived upright orientations of these nonrepresentational forms are the results of general experience with forms in the everyday world. To the young child, forms would be essentially neutral in orientation, but by repeatedly experiencing forms in their normal, upright orientation, the child begins to associate certain features with the normal orientation so that these features would come to stipulate uprightness. Insofar as the children had similar perceptual experiences during this period, they would come to agree increasingly with one another on the judged upright orientation of forms.

This developmental change, however, has not been found consistently. Braine also found that the initially high rate of agreement among her 4- and 5-year-olds nearly vanished among the 7- and 8-year-olds, while we found (Harris \& Schaller, 1971) that agreement among 7-and 9-year-olds was slightly stronger than that among 5- to 6-year-olds. We elsewhere have suggested that certain features of Braine's design may have made the task confusing to older children (Harris \& Schaller, 1973), and if we are correct, then there may be no true decline in strength of agreement. If there is a decline, however, then it must be explained. Perhaps, after long experience with forms (so that even the most uncommon orientation has been experienced often), the strength of the associations to the cues might become similarly strong for other orientations. Thus, the tendency toward choosing one orientation would drop out, and agreement would decline with age. ${ }^{7}$

Whether agreement declines with age or not, both the focal point and experiential hypotheses hold that a variety of forms specify the upright orientation, even to the young child. The main concerns of the current investigation were: are there general features intrinsic to the form itself that constitute the basis for the judgment of upright orientation? If so, what are these features?

Howard and Templeton (1966, p. 295ff) have suggested that the perceived uprightness of forms depends on three types of axes that might be found in a form-(1) the axis of symmetry. (2) the axis of the main lines of the form, and (3) an axis between significant landmarks in the forms. There must be other features that also contribute to upright judgments. Our aim was to make the first systematic attempt to assess the possible effects of some of these intrinsic features on the adjudged upright orientation of two-dimensional forms, and to compare those effects across age. In so doing, we can compare the "focal-point" and "experiential" hypotheses described above. Our procedure was to have subjects of different ages set "upright" forms that systematically incorporated a variety of features, in different combinations, that seemed likely to underlie orientation judgment. The features used are listed here.

A form having any identifiable upright orientation would seem to have to possess at least one of the following three types of axes:

Axis of symmetry. An imaginary line drawn in the plane of the form around which the form would be symmetrical. This seems, on the basis of our earlier work and analysis by others, to be an important determinant of adjudged orientation.

Main lines. The longest lines (straight or nearly so) in the border or outline of the figure. This definition differs from Howard and Templeton's (p. 296), which includes what here shall be called "texture lines." Ordinarily the longest straight sides would be the predominant main lines.

Texture lines. Lines or other indications of texture that have a predominant direction and lie inside the form, as distinct from main lines which lie on the border of the form. For example, a ladder's main lines 
run perpendicular to its texture lines.

These three features can specify only the "up-down" alignment of a form. Other features would have to specify which end is "up," and which "down." These include:

Polar axes. The axes running between different and "significant landmarks in the forms" (Howard \& Templeton, 1966, p. 296). An example would be a circle at the end of a straight line.

Texture density gradient. Different spacing of texture lines, with a gradient in one direction, e.g., apparent closer spacing of railroad ties in the distance.

Taper. Gradual approach, or convergence, of the main lines (outlines) of the form, from one end to the other, e.g., apparent convergence of railroad tracks in the distance. Howard and Templeton (1966, p. 298) suggest that things that do not taper toward the top tend to look "off-balance," though there are exceptions in everyday life.

Weight. The largest area of visual mass, by virtue of the largest area or the greatest number of features.

Light-dark opposition or gradient. Predominantly lighter areas opposed to or grading into predominantly darker areas. Gibson (1966) has pointed out that, in the outdoor visual field, the rule is radiant light from above, reflected light from below. The tops of things, therefore, tend to be lighter than the bottoms.

Stable base. The flat side on the base or bottom for flat-sided forms; for forms lacking a flat side, the side or sides on which the form could most easily balance on a flat surface.

\section{METHOD}

\section{Materials}

Two sets of forms were used. Set 1 included 23 forms designed to test the various hypotheses of the bases of orientation judgment. Most of these forms incorporated more than one of the features named above and several represented variations on a basic design.

Set 2 included 15 forms designed by Braine (Ghent, 1961) and used by Harris and Schaller (1971, Note 4); for comparison, this second set was shown to half the subjects in addition to Set 1 . In addition, for purposes of another study, the remaining subjects viewed a third set of 12 forms (which will not be discussed in the present report. $)^{8}$

Each form was drawn in black on white and photographed for slide presentation. Each slide, when projected, showed a white circular ground with the form inside. The circle was approximately $20 \mathrm{~cm}$ (8 in.) in diam and subtended an angle of approximately $4^{\circ} 44^{\prime}$ when projected $2 \mathrm{~m}$ from the subject.

\section{Apparatus}

The forms were projected by a slide apparatus and mirrors so designed that the subject could rotate the slide to any desired orientation by turning a $43-\mathrm{cm}$ wheel in front of him. The projected image turned in exact correspondence to manipulation of the wheel. The apparatus allowed free choice of orientation over the entire $360^{\circ}$ range so that responses would not be biased toward only a few categories (cf. Harris \& Schaller, 1971).
The forms were projected onto a flat white rectangular cloth screen $2 \times 3 \mathrm{~m}$ in dimension, suspended with the edges vertical and horizontal. The screen was sufficiently larger than the projected image so that the screen borders would not unduly influence the judged orientation of the form. ${ }^{9}$ Illumination was dim and diffuse from behind the subject, thus eliminating visible texture cues from the screen.

\section{Subjects}

A total of 80 subjects viewed the figures, with 16 subjects ( 8 boys) at each of five grade levels: Kindergartners, first-, third-, and fifth-graders from a public school, and summer-session college volunteers (who had a wide age range). The mean age for each group was: kindergarten, 6.1 years; 1 st, $7.0 ; 3 \mathrm{rd}, 9.3$; Sth, 11.2; and college, 27.3.

\section{Procedure}

Each subject was tested individually (the grade-schoolers in school, the college students in the psychology building). For the younger children, the experimenter's instructions were as follows: "I'm interested in how boys and girls can know which way things are right-side-up, the way they're supposed to be. If I ask you which way these toys should go, you'd know, wouldn't you? Here, put them the way they're supposed to be, so they're right. [The experimenter presented a toy truck and a small plastic lion, both on their sides. All the subjects immediately set them aright.] OK. Good. You know. Now what about this picture? [A picture of a child's face came on the screen.] This machine lets you move the picture any way you want. [The experimenter turned the apparatus wheel in both directions, ending with the face on the screen in some nonvertical orientation.]. There, you try it. [The subject played with it for a few seconds; several subjects spontaneously turned the face right side up. If this happened, the experimenter, as he spoke, turned the fact so it again was obliquely oriented.] He looks pretty funny, that way, doesn't he? [Subjects agreed.] Make him the way he should be. Put him right. [All subjects performed quickly and correctly.] OK. Good. Now, I've got some other pictures here and I want to see if you can do the same thing. OK? You look at each picture and turn it until you think it's just right, the way it's supposed to be, just as you did with the boy and the truck and the lion. OK? Here's the first one. You turn the wheel until you think it's just the way it's supposed to be, so it's just right."

The toy sequence was omitted and the instructions shortened for the fifth-graders and college students.

Responses were recorded to the nearest $5^{\circ}$, as indicated by a scale hidden on the back of the apparatus. Each subject was told, when the session was over, that he had done very well.

The forms were presented in blocks, two to each child, with the Braine forms comprising Set 1 (16 forms), the newly designed forms, Set 2 (23 forms), plus a third set of 12 forms. ${ }^{8}$ The sets of slides were presented in four sequences: Sets 1 and 2,2 and 3, 2 and 1 , and 3 and 2 . One quarter of the subjects (counterbalanced) saw each of the sequences. Thus, 40 subjects oriented the Braine (Ghent, 1961) forms; all 80 subjects oriented the new forms.

\section{RESULTS AND DISCUSSION}

\section{Analysis}

The Kolmogorov-Smirnov goodness-of-fit test was the main statistical test used. ${ }^{10}$ Tests were performed for each form separately within each grade and over all grades to determine whether significant sex or order-of-presentation differences existed, and to compare each distribution against a random distribution. Additional comparisons were made to identify grade (age of subject) effects. 
Table 1

Forms as They Were Judged to be Upright by $10 \%$ or More of the Subjects in Grade Groups

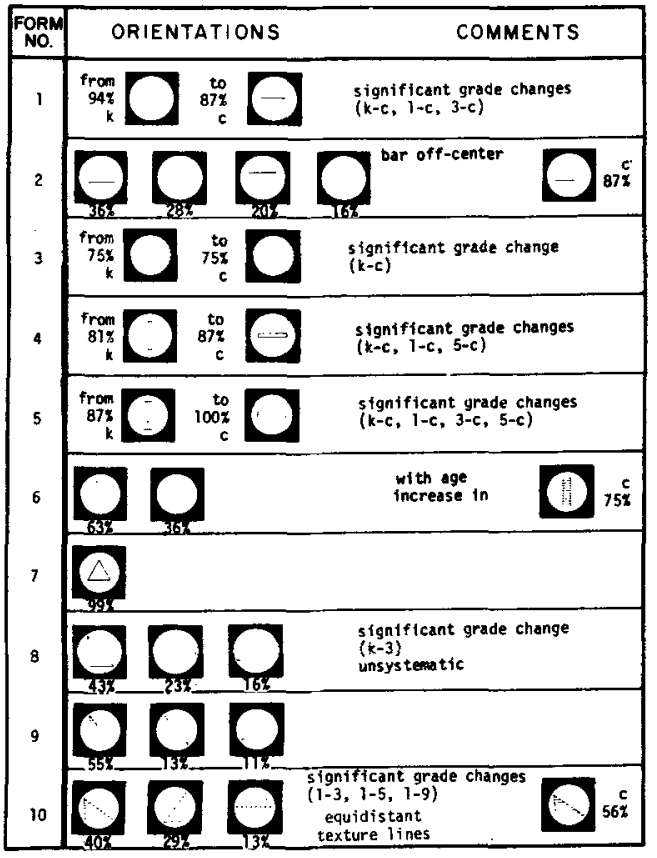

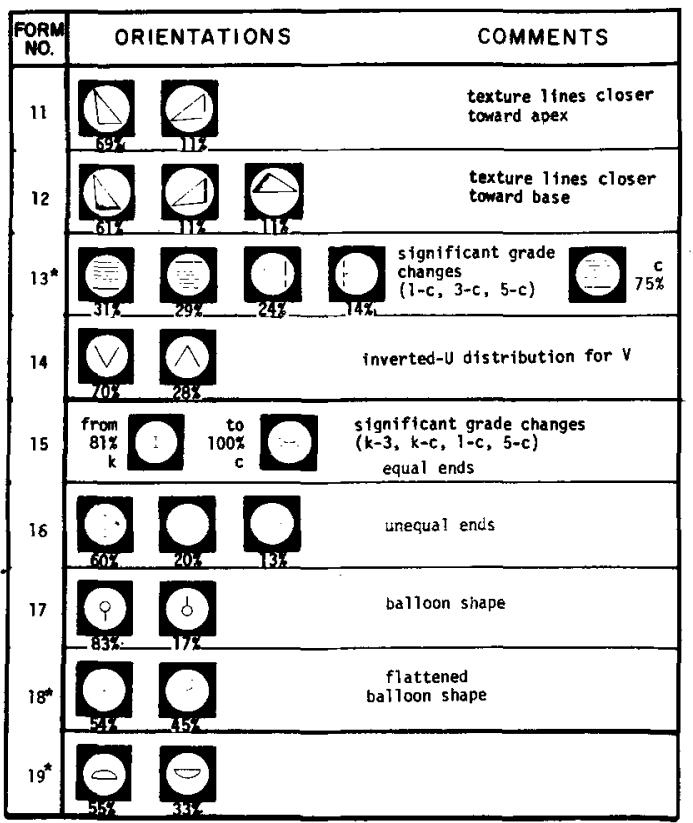

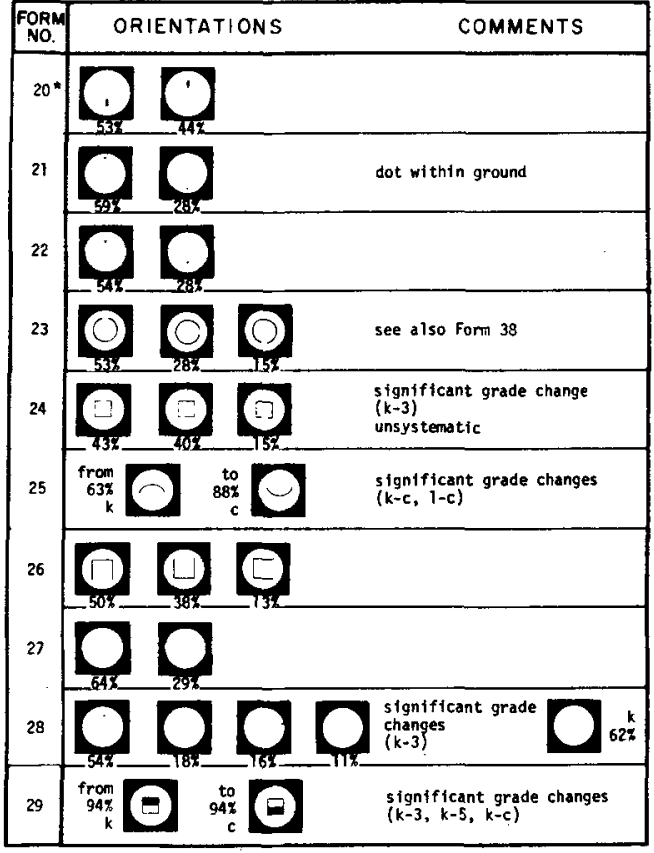

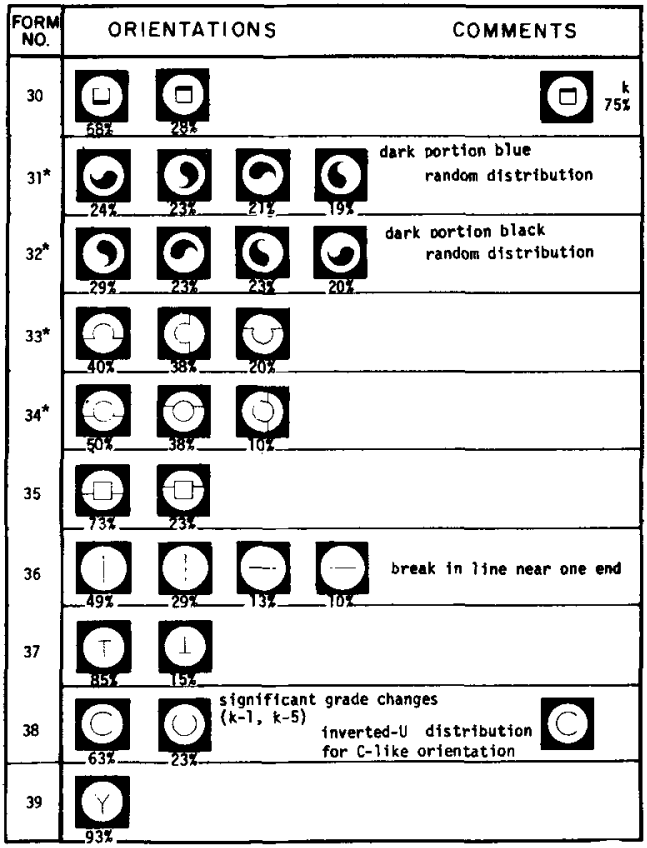

Note-The forms are displayed in one of two formats: (I) For forms for which there are significant differences between grades in choice of the upright orientation. the direction of these differences is illustrated by showing the orientation selected by the majority of each of the two extreme age groups (kindergartners and college students). The percentage of these two grade groups choosing the orientation shown is given beside the form in the "from ... to" captions. Grades that differed significantly in distribution of orientation judgments are indicated under "Comments" in parentheses $(k=$ kindergarten, $1=$ first grade, $3=$ third grade, $5=$ fifth grade. $c=$ college). (2) For forms for which there were no significant differences in the distributions of upright judgments between grade groups. judgments from all groups taken together are shown. Each of the orientations of the form made by $10 \%$ or more of the subjects is shown, with the percentage of subjects in all grade groups selecting that orientation written below the form. Asterisks in Column 1 indicate forms for which the placements of the forms into the categories shown did not constitute a significant choice among the displayed orientations. 
Only 3 of the 255 within-grade comparisons on the basis of sex of subject yielded significant $(p<.05)$ effects. There were no significant sex effects over all grades taken together for any of the 51 forms. Order differences were also negligible (18 out of 1,530 contrasts were significant, and in no systematic way). Therefore, data for all sex and order conditions were combined for the main analyses.

The tests for systematic groupings $\left(\mathrm{H}_{\mathrm{o}}\right.$ is a uniform distribution) disclosed two figures for the grades combined whose groupings were insufficiently consistent for the test to distinguish from random distributions of orientation. These were Forms 31 and 32 , the modified yin-yang symbols. Inspection of the distributions disclosed that there indeed were groupings of the orientation judgments, though in some cases dispersed widely over about $30^{\circ}-40^{\circ}$ on either side of the cardinal points. ${ }^{11}$

For several new forms, adjudged upright orientation changed systematically with grade, with the orientation most chosen by the kindergartners and younger children yielding progressively to the most-chosen orientation of the adults. These changes will be discussed for individual forms.

For forms not showing systematic grade changes, chi-square tests were performed to determine whether one category was chosen significantly more often than others (excluding categories with less than $10 \%$ of judgments). Of the new forms and Braine forms, eight (marked in Table 1 with an asterisk) showed no significant difference among choices of the categories.

\section{Individual Forms}

Table 1 shows the forms in orientations chosen by at least $10 \%$ of the subjects. Significant grade differences are noted. The forms are shown within groups of similar or related forms, rather than in the order of presentation to the subjects. ${ }^{12}$

The forms are displayed in two formats: as noted, for several forms (e.g., Form 1), there were significant differences between grades in placement of upright orientation; for these forms, direction of these differences is illustrated by showing the orientations selected by the majority of the kindergartners and by the majority of the college students. In nearly all of these cases, the changes are very orderly progressions from kindergarten through the middle grades to college. Thus, to say that the orientation shifted with grade is not misleading. These changes appear mostly to involve changes in the orientation of main-line axes or texture lines, with younger subjects aligning these cues vertically, older subjects, horizontally.

For other forms, for which there were no systematic grade changes in distribution, a second format was used-each placement is shown with the percentage of all subjects across grade placing the form upright in that orientation. An example is Form 6.
The regular systematic changes in the adjudged upright orientation of the new forms that occur with grade are surprising. One would expect, on the basis of Braine's study with 4- to 8-year-olds (Ghent, 1961), that strength of agreement would weaken with age, or, on the basis of Harris and Schaller (1971), that strength of agreement would remain constant. Instead, there is a shift from one agreed orientation at the younger grades to another at the college level, with stronger agreement at the college leve. The change is gradual, creating mixed agreement in the middle grades. The judgments for Form 1 typify this shift. The youngest children in both Braine's study and the current study oriented this form (a straight line centered in the circular ground) with the line vertical. As Figure 1 shows, however, across school grade the children increasingly tended to orient this form horizontally, until by college age the agreement is nearly as strong $(87 \%)$ for the horizontal orientation of Form 1 as it is for the vertical in the kindergartners $(94 \%)$. The same progression occurs with the other "elongated" forms (refer to Table 1 again), for example, a plain rectangle (Form 4), a rectangle with texture lines running in the same direction as the main lines (Form 5), a "dumbbell" shape with equal circles on both ends (Form 15), and parallel lines alone (Form 3). Most kindergartners placed all these forms vertically; most college students placed them horizontally.

\section{Polar Axes}

The age progression from vertical to horizontal orientation occurred only for new forms with two axes of asymmetry, that is, lacking a polar axis. In Forms 16 or 17, for instance, or in Form 20 (again a bar but now with distinctive features at one end; see Table 1), horizontal orientations occur very infrequently, and even for the college students the two vertical orientations predominate. Thus, within the constraint that the axis of symmetry remains vertical, orientation judgment is radically influenced by polar features.

\section{Texture Lines}

Adding texture lines to the form either strengthened or weakened the "main line" effect depending on whether the lines run in the same direction as or opposite to the main lines of the figure. For Form 5, with the texture lines congruent with the main lines, the younger children chose the vertical, the college students chose the horizontal, with both age groups choosing more consistently with this form than with the plain rectangle. When the texture lines were incongruent with the main line orientation, the reliability of the younger children's "vertical" choice was disrupted and, over age, responses changed consistently, culminating in a new vertical choice for the college students. Apparently the texture lines can 


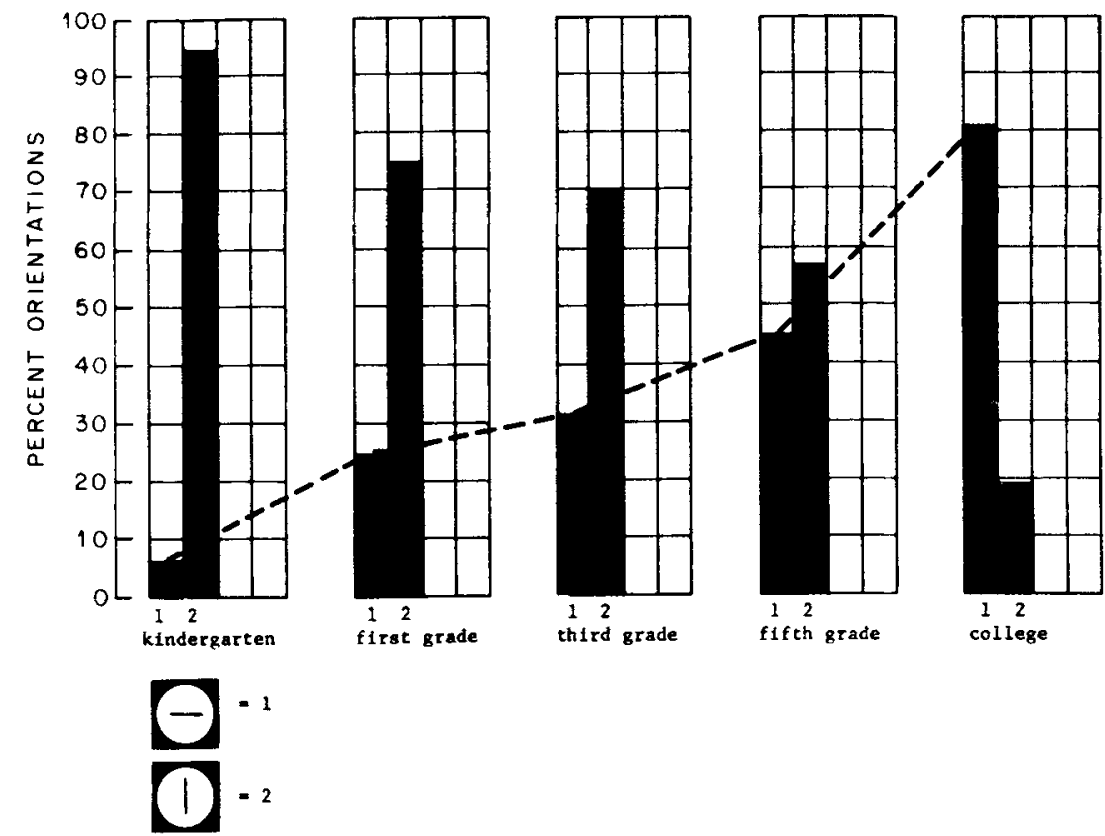

Figure 1. Distribution of judgments of upright orientation for Form 1, a bar centered in the ground. Most frequent judgment moved from vertical alignment for kindergartners to horizontal aligmment for college students. Similar progressions with grade occurred for the other elongated forms. Increase in the "adult-like" orientation is shown by the dashed line. outweigh the main lines in determining the adjudged upright orientation.

\section{Triangles}

The process of strengthening or disruption also occurs among the scalene triangles. When the texture lines are horizontal (Form 9) in the most frequently chosen orientation of the basic figure, the choice of the "basic" orientation is increased. When the texture lines run vertically (Form 10) in the "basic" orientation, choice of the "basic" orientation is weakened, and the orientation with the texture lines horizontal, previously chosen by only $16 \%$ of the subjects as upright (cf. Form 8, Orientation 3), is now chosen by $40 \%$ of the subjects (Form 10, Orientation 3).

\section{Texture Density}

Forms 11, 12, and 13 were designed to test the effect of texture density changes. Form 11 has texture lines which become closer toward the "tip" of the form, in a manner consistent with the closer visual spacing of more distant natural elements on the earth's surface, i.e., with the "natural" texture density gradients. In Form 12, on the other hand, the texture density changes in the opposite direction. On the basis of a "natural texture density gradient" hypothesis, the selection of Orientation 1 would be expected to increase in frequency from Form 9 to Form 11, and to decrease in Form 12. The obtained percentages were $55 \%, 69 \%$, and $61 \%$, respectively. While the percentage for Form 11 is in the expected direction, that for Form 12 is not and any effect is weak. Perhaps both increases are the result of more texture lines rather than a texture density effect.

In addition, for the younger grades, there is mixed performance on Form 13, a "square array" of texture gradient lines. For the college group, however, there is a clear difference, with choice of the horizontals reaching nearly $100 \%$ and the orientation with greater density at the bottom accounting for $75 \%$ of the responses, in contradiction to a texture density hypothesis. This evidence, together with that from the triangles (Forms 11 and 12), therefore, fails to support a texture density hypothesis. Perhaps the lines instead are cues for "weight," predisposing location at the bottom.

\section{Light-Dark Differentiation}

Forms 29, 30, 31, and 32, each having one major white and another dark or colored area, were designed to test the effect of relative darkness in one part of the figure. Forms 31 and 32 (yin-yangs) were chosen because they included few, if any, other orientation cues. The light-dark differential (with "light-at-top") is the only cue expected to operate. For these two forms, there was no statistically detected difference in the frequency of choice of any orientation. The pattern for the square "light-dark" forms (29 and 30) is inconsistent across studies. Braine's (Ghent, 1961) results would indicate $75 \%$ choice of black-on-top as upright for both forms with no clear change across age (4 to 8 years). In our previous study (Harris \& Schaller, 1971), the pattern for these same forms was weak and inconsistent at all ages ( 5 to 9 years). Now, in the present study, $75 \%$ of the kindergartners chose 
black-on-top for the quarter-black square (Form 30), consistent with Braine's findings. But of the remaining subjects, $68 \%$ chose white-on-top. For the half-black square (Form 29), this suggested age change was far clearer: $94 \%$ of the kindergartners chose black-on-top as upright, and $94 \%$ of college students chose white-on-top. If any consistent tendency can be seen in the three studies, it is for choice of black-on-top for the younger children and choice of white-on-top for the older children and adults.

While the older subjects' judgments are consistent with the hypothesis that sky-at-the-top or light-on-top is an intrinsic orientation cue, the kindergartners' judgments are damaging to it. The results must mean that dark-at-top is the earliest cue for upright orientation, and that perhaps with experience light-at-top outweighs that tendency. Light-at-top as a cue can easily be viewed in terms of environmentally consistent cue learning, but we have no explanation for the finding with the young subjects.

\section{Letter-Like Forms}

Placements of several of the apparently letter-like forms reflect the effect of letter resemblance on orientation, and again, as we found earlier (Harris \& Schaller, 1971), the distribution of placements for some forms is inverted U-shaped, rather than linear. ${ }^{13}$ For forms which clearly are letters, such as the $\mathrm{Y}$ and $T$, there is consistent letter-consonant orientation across age. For others, however, which are merely letter-like, the letter-consonant orientation first increases, then decreases (see Figure 2). As we have written elsewhere (Harris \& Schaller, 1971, Schaller \&
Harris, 1974), such results suggest that as children begin to recognize letters and letter-like forms and as their competence with letters increases, they are more likely to orient letter-like forms in letter-like orientations. But as the children's competence continues to grow, forms that merely resemble letters are no longer good enough, and are now rejected as letters and are placed in orientations other than letter-consistent ones.

\section{Focal-Point Hypothesis}

Finally, Forms 16, 17, 18, and 20 vary the features placed at the polar ends of the forms and therefore bear on Braine's hypothesis that the form will be seen as upright when the focal point is at the top (Ghent, 1961). For Form 17, our previous findings (Harris \& Schaller, 1971) concurred with Braine's, and the current study also finds significantly greater choice of the balloon-like orientation across all grades.

Since previous results with this balloon-shape supported a focal point hypothesis, the shape was modified to further test the hypothesis. For instance, when the balloon shape is given a flattened top (Form 18), orientation placements change sharply. The previously strong agreement shown for the balloon (Form 17) vanishes, and slightly more subjects now orient the form (Form 18) with the circle on the bottom as if resting on the flattened end. Form 20, a line with a block at one end, shows the same results. Braine's subjects agreed almost totally that Form 20 was upside-down with the weight at the bottom, whereas most of our subjects placed it right-side-up this way. There are no significant grade differences for either of these forms. Finally,

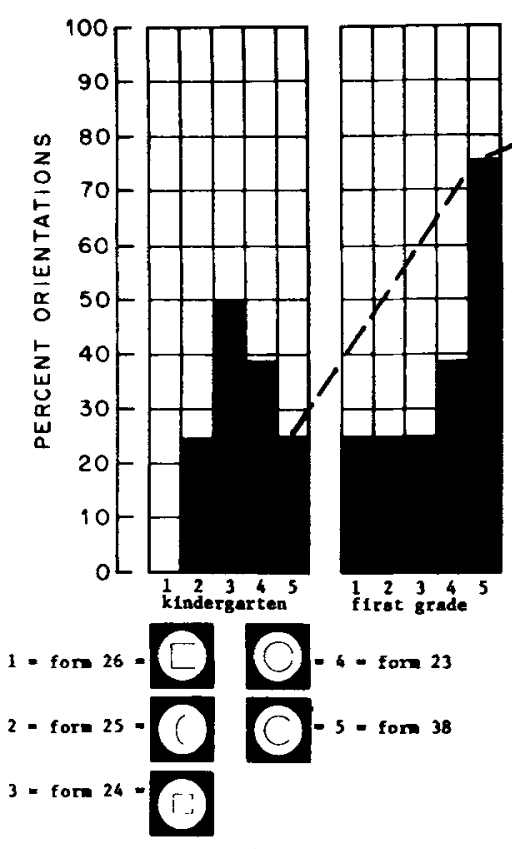

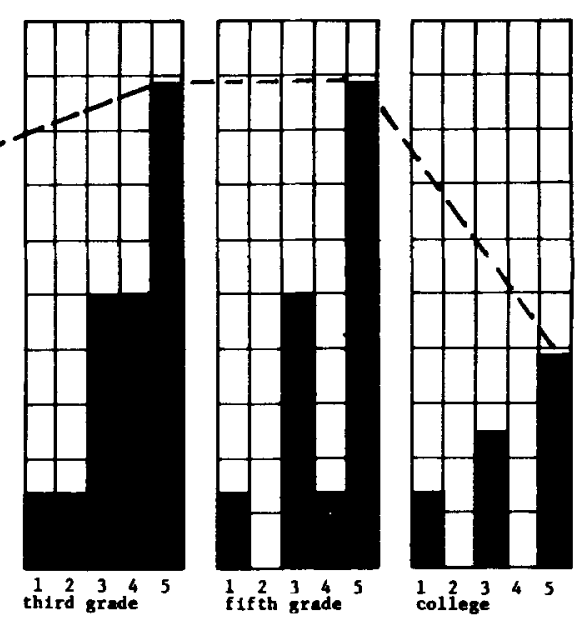

Figure 2. Distribution of judgments of upright orientation in "facing-right" orientation of several forms judged a priori to resemble in increasing order the English letter "C." The ordering is based on Harris and Schaller, 1971. For each form, there is elther a decrease of C-like judgments with grade or an increase followed by a decrease with grade, as for Forms 23, 26, and 38. The shape of the distribution of $\mathbf{C}$-like judgments for the most C-like form, Form 38, is indicated by the dashed line. 
Form 16, the unequal dumbbell, is oriented significantly often with the polar and symmetrical axis vertical, with the greater weight at the bottom.

Each of these modifications of the balloon shape would seem to accentuate the focal portion of the form and therefore the focal-point prediction ought to have held. In their recent review, Pick and Pick (1970) have suggested apparent exceptions to the focal-point hypothesis; in some cases, forms with obvious focal points do not obey the rules (as here); in other cases, there is no reasonable focal point (in many cases we have found it difficult to specify where the focal point is). Nevertheless, consistent judgments of orientation are made. The current results well illustrate these points. Perhaps "focal point" should be considered epiphenomenal to the basis of orientation; that is, to speak of a focal point is to speak of one or, more likely, a collection of separate and distinct intrinsic features of a form. And it is these features in combination with other factors such as scanning tendencies, letter resemblance, or resemblance to common objects, in different combinations depending on the age of the subject, that make a form look upright in a particular orientation.

\section{Responses to Intrinsic Features: Summary}

Though the forms used here were not entirely systematic in their incorporation of possible orientation cues, we nevertheless can assess the relative strength of each cue and compare cases in which one cue seems to outweigh another.

One of the strongest bases of judgment, which, moreover, appears to remain constant over age, is the axis of symmetry or greatest symmetry. For $97 \%$ of the forms having an axis of symmetry (including greatest symmetry), the forms were made upright most often in the orientation that aligns the axis of symmetry vertically. The single exception was the C-like form (No. 38). Comparison with the other predictive bases shows that vertical symmetry is by far the most consistent predictor of axis of orientation, although, as we said, the cue cannot specify which end is up and which down.

It is reasonable, from an "experiential" point of view, that vertical symmetry is a strong basis for form orientation, since, as Howard and Templeton (1966) point out, it is difficult to find in a normal room an object that is not bilaterally symmetrical and aligned with the plane of symmetry vertical. Perhaps some aspect of engineering practice accounts for this consistency, and then subsequent experience with the consistent orientation of everyday objects becomes the basis for the perception of a symmetrical object as upright only when vertically aligned. But, in the view of the strength of this effect in the grades tested by Braine and by ourselves (and for children in other cultures; Antonovsky \& Ghent, 1964; Serpell, 1971) and in light of the frequency with which symmetry is known to affect performance in perceptual tasks (e.g.,
Rock \& Leaman, 1963; Zusne \& Michels, 1962), it may be more likely that the consistency of orientation judgments shown here is accounted for by some interaction between symmetry of form and a basic, perhaps unlearned, characteristic of the visual system. For example, there may be receptive field specialization in humans for perception of edges in particular alignments (cf. Appelle, 1972; Leibowitz \& Harvey, 1973; Sekuler, 1974).

The main line axes of the forms are associated with less consistency of judgment than the symmetry axis because of the shift with age from vertical orientation by the young children to horizontal orientation by the adults. Thus main lines are oriented vertically (the predicted orientation) in only $31 \%$ of the relevant cases, mostly by children. By adding to these cases the adults' consistently horizontal judgments, the age-modified agreement scores greatly increase. In the few cases where main line axis is opposed to axis of symmetry, symmetry seems to be the stronger cue (e.g., Form 34).

The same shift with age from vertical to horizontal orientation occurs for the texture lines. Perhaps the adult response to these "natural" cues for horizontal orientation must actually be learned through long experience. The young children may regard the lines merely as more main lines.

The polar axis is usually coincident with the main line or the symmetry axis in the forms used here, so it is difficult to distinguish the effect of a polar axis. Of the relevant forms, $79 \%$ were generally aligned with the polar axis vertical.

"Stability" seems to provide a somewhat reliable basis for prediction $(68 \%$ of the relevant cases seem stable), especially if there is a flat bottom to the form.

Other features have somewhat less clear effects. Taper, for instance, appears to operate fairly regularly in the triangles, predisposing taper toward the top. Those negative instances of taper-to-top are forms resembling letters. In fact, the letter-like quality seems to outweight all the other factors, even vertical symmetry, as shown, for example, by the results for the C-like Form 38 .

In summary, the axes of bilateral symmetry, axes of main lines of the forms, and axes between polar features of the forms seem strongly associated with the adjudged upright orientation. Resemblance to some commonly experienced forms, in the current case letters of the alphabet, may outweigh intrinsic features of the forms in determining orientation. The focal-point hypothesis fails to consistently predict the upright orientation in the tested cases. Finally, features intrinsic to the forms, such as flatbottomedness or taper, contribute to judgment of the upright orientation.

\section{Developmental Changes: Summary}

A number of striking changes in orientation of the forms occur with increasing grade level. The changes 
appear to be orderly and consistent between forms. It is also clear that the degree of agreement among college subjects exceeds that for the kindergartners and that the 'kindergartners' exceeds all other children. This developmental effect is greatest for the newly designed forms, with the 15 Braine forms showing no significant grade effects. The average of the highest percentage of choice for each new form for the kindergartners through college groups, respective$\mathrm{ly}$, are $60.1 \%, 54.5 \%, 58.0 \%, 54.6 \%$, and $70.5 \%$. A chi-square test on the frequencies indicates significant differences among grades. The weaker concordance among subjects in the 1st, 3rd, and 5th grade groups (in conjunction with examination of the age effects for individual forms) indicates that the bases for judgments of these forms are changing throughout the interim grades. The basis for perception of the upright is relatively more stable at the kindergarten level and is again stable by the time the college level is reached. The greater agreement among the college students, despite their more variable age, seems to indicate that the final basis for perception or judgment of the upright is relatively uniform and long-lasting. Thus-in place of the proposed hypothesis that experience with forms in all orientations causes a weakening of agreement with age-the simplest explanation would seem to be that both children and adults can strongly agree which way things are up, though their choices differ.

These age changes were unexpected and point up the need in studies of this kind for an age range great enough to allow a determination of the total developmental trend (cf. Wohlwill, 1960). An extended age range, perhaps even greater than included here, is particularly needed in studies of orientation judgment, in light of the current results-reversals of agreed judgment, inverted-U distribution, and in some cases changes with age from no agreement to agreement.

The change from one strongly chosen orientation for the young children to another equally strongly held choice for the college students is puzzling. Braine's (1972) hypothesis that the change with age is from one top-to-bottom strategy to a multiplicity of strategies predicts a decline with age in strength of agreement of judged orientation. It does not predict what we have found-a shift in direction of agreement. Most of the developmental changes occur in forms that are elogated in some way. These might be the forms in which general sequential processing and preference for orientation of the type Braine proposes in her focal point hypothesis are operating most powerfully because of the strong linearity of the forms. Thus it would make more sense with these forms than with others to invoke a process of scanning from one end to the other.

A possible explanation of this finding, therefore, is that the changes in judgment represent different patterns of preferred processing between the children and adults. Braine might be correct, in part, in the specific supposition that children generally process from top to bottom, or at least vertically. Then, for young children, the agreement on vertical orientation could be due to tendencies of the type Braine suggests. Adults, if their preferred processing patterns were instead from left to right, as in reading, might then orient the forms horizontally. Alternatively, the children's agreement could reflect an innate preference for verticals, which could be modified by adults' substantial experience, including training in left-to-right (horizontal) scansion through reading. However the change in orientation is explained, our argument about form linearity would predict that strongly linear forms, i.e., forms that deviate from squareness or roundness, would be judged by children to be upright when vertical, but would be judged by adults as upright when horizontal. For most other forms, orientation judgments would not be expected to change significantly with age.

\section{REFERENCES}

Appelle, S. Perception and discrimination as a function of stimulus orientation: The "oblique effect" in man and animals. Psychological Bulletin, 1972, 78, 266-278.

Antonovsky, H. F., \& GHent (Braine), L. Cross-cultural consistency of children's preferences for the orientation of figures. American Joumal of Psychology, 1964, 77, 295-297.

Braine, L. (GhENT). Age changes in the mode of perceiving geometric forms. Psychonomic Science, 1965, 2, 155-156.

BRAINe, L. (GHENT). A developmental analysis of the effect of stimulus orientation on recognition American Journal of Psychology, 1972, 85, 157-187.

Brooxs, R. M., \& Goldstein, A. G. Recognition by children of inverted photographs of faces. Child Development, 1963, 34, 1033-1040.

DAvidson, H. P. A study of reversals in young children. Pedagogical Seminary and Journal of Genetic Psychology, $1934,45,453-465$.

Davidson, H. P. A study of the confusing letters $b, d$, p, and $q$. Pedagogical Seminary and Journal of Genetic Psychology, 1935, 47, 458-468.

Ghent, (Braine), L. Recognition by children of realistic figures presented in various orientations. Canadian Journal of Psychology, 1960, 14, 249-256.

Ghent, (Braine), L. Form and its orientation: A child's-eye view. The American Journal of Psychology, June 1961, 74, $177-190$.

Ghent (Braine), L., \& Bernstein, L. Influence of the orientation of geometric forms on their recognition by children. Perceptual and Motor Skills, 1961, 12, 95-101.

Gieson, E. J., Gibson, J. J., Pick, A. D., \& Osser, H. A developmental study of the discrimination of letter-like forms. Journal of Comparative and Physiological Psychology. $1962,55,897-906$.

Gibson, J. J. The senses considered as perceptual systems. New York: Houghton-Mifflin, 1966.

Goldstein, A. G. Learning of inverted and normally oriented faces in children and adults. Psychonomic Science, 1965, 3. 447-448.

Goldstein, A. G., \& Chance, J. E. Recognition of children's faces. Child Development, 1964, 35, 129-136. 
Goldstein, A. G., \& Chance, J. E. Recognition of children's faces: II. Perceptual and Motor Skills, 1965, 20, 547-548.

Harris, L. J., \& Allen, T. W. Role of object constancy in the perception of object orientation: Some methodological considerations in studies of human infants. Human Development, 1974 , $17,187-200$.

HaRris, L. J., \& Schaller, M. J. Form and its orientation: Re-examination of a child's-eye view. American Journal of Psychology, 1971, 84, 218-234.

HarRis, L. J., \& Schaller, M. J. Reply to Braine: Reexamination of a child's-eye view. American Journal of Psychology, 1973, 86, 655-660.

HaYs, W. L. Statistics for psychologists. New York: Holt, Rinehart, and Winston, 1963.

HoWARD, I. P., \& TEMPLETON, W. B. Human spatial orientation. New York: Wiley, 1966.

KofFKA, K. The growth of the mind. New York: Harcourt Brace, 1924.

Leibowitz, H. W., \& Harvey, L. O. Perception. In P. H. Mussen and M. R. Rosenzweig (Eds.), Annual review of psychology (Vol. 24). Palo Alto: Annual Reviews, 1973.

Pick, H. L., \& Pick, A. D. Sensory and perceptual development. In P. H. Mussen (Ed.), Carmichael's manual of child psychology (3rd ed.). New York: Wiley, 1970.

Rock, I., \& Leaman, R. An experimental analysis of visual symmetry. Acta Psychologica, 1963, 21, 171-183.

Schaller, M. J. The role of intrinsic features in the judgment of 'upright' orientation of two-dimensional forms: A developmental analysis. Doctoral dissertation, Michigan State University, East Lansing, 1972.

Schaller, M. J., \& Harris, L. J. Children judge "perspec tive" transformations of letter-like forms as different from prototypes. Journal of Experimental Psychology, 1974, in press.

Sekuler, R. Spatial vision. In P. H. Mussen and M. R. Rosenzweig (Eds.), Annual review of psychology (Vol. 25). Palo Alto: Annual Reviews, 1974.

SERPELl, R. Preference for specific orientation of abstract shapes among Zambian children. Journal of Cross-Cultural Psychology, 1971, 2, 225-239.

SIEGEL, S. Nonparametric statistics for the behavioral sciences. New York: McGraw-Hill, 1956.

STERN, W. Psychology of early childhood (English trans., 3rd ed.). New York: Holt, 1930.

Worwill, J. F. Developmental studies of perception. Psychological Bulletin, 1960, 57, 249-288.

WoHLwill, J, F., \& WeINER, M. Discrimination of form orientation in young children. Child Development, 1964, 35, 1113-1125.

WRIGHT, D. M. Development of recognition memory for forms, letters, faces, and tools as a function of orientation. Doctoral dissertation, University of Wisconsin, 1974.

Zusne, L., \& Michels, K. M. Geometricity of visual form. Perceptual and Motor Skills, 1962, 14, 147-154.

\section{NOTES}

1. Goldstein, A. G. Developmental changes in recognition of inverted stimuli. Paper presented at the Biennial Meetings of the Society for Research in Child Development, Philadelphia, Pennsylvania, 1973.

2. Wright, D. M., \& Schaller, M. J. Development of recognition memory: Orientation and type of form. In preparation.

3. Schaller, M. J. Age-related changes in recognition memory of faces: No peak at 16 years. In preparation.

4. Harris, L. J., \& Schaller. M. J. Preference vs. upright orientation of various two-dimensional forms. Paper read at the
Biennial Meetings of the Society for Research in Child Development, Minneapolis, Minnesota, 1971.

5. Schaller, M. J., \& Dziadosz, G. M. Asymmetries of visual recognition in the central four-degree field by adults. In preparation.

6. Schaller, M. J., \& Dziadosz, G. M. Asymmetries of visual recognition in the central four-degree field by children. In preparation.

7. Still a third possibility remains-that certain features specify orientation a priori, that is, without necessity of past experiences. There is no way to address this possibility in this research. This kind of model could still be compatible with a decline in strength of agreement, if one supposed that with increasing experience with forms in various orientations other than the upright the initial specification of orientation would be weakened.

8. The forms were the 12 standard forms from the Gibson, Gibson, Pick, and Osser (1962) and the Schaller and Harris (1974) studies of the discrimination of letter-like forms. They were included in the preliminary analyses in the current study, but, as might be expected for forms not designed with orientation in mind, they did not elicit strong agreement of upright orientation judgments.

9. By frame cues, we mean portions of the form or ground which move with the form as it is rotated, as in Braine's (Ghent, 1961) procedure, where the square edges of the card rotated with the form. By frame cues, we do not mean edges or lines or walls in the room such as doors or tables which remain in a constant orientation despite rotation of the form. These latter room cues are the reference in relation to which the subject orients the forms. They are not, however, the basis on which the subject orients the forms.

10. The nonparametric statistics (after Siegel, 1956) programs of the Computer Institute for Social Science Research at Michigan State University were used to perform the tests. The Kolnogorov-Smirnov one-sample test tests good ness-of-fit between a cumulative distribution of a set of sample values and some specified theoretical multicategory distribution; the two-sample test. between two sample distributions to see if they could reasonably be regarded as having come from the same population. According to Siegel (1956), Hays, (1963), and other statisticians, the tests are more appropriate than chi square for small samples. All scores were transformed so that for certain figures, e.g., the equilateral triangle or rectangle, equivalent orientations were treated as the same. In addition, before the tests were carried out, the beginning of each distribution was moved from the arbitrary zero point $\left(0^{\circ}\right.$ from reference orientation) to a point higher in the distribution where very few orientations fell. This procedure precluded obtaining significant differences based on a difference between scores near $5^{\circ}$ and $355^{\circ}$, which, though physically very similar, would otherwise be analyzed as coming from opposite ends of the distribution.

11. We point out, in passing, that the conclusion drawn in our earlier study (Harris \& Schaller, 1971, 1973) again holds, namely, that presentation of the forms in only two orientations (as in Braine's procedures) would significantly constrain the subjects' range of choices for a number of forms. However, the results indicate that the subjects' choices of orientations for most forms would not have been significantly limited had the forms been presented in from two to four orientations specially selected through pretesting (not always the four "cardinal" points).

12. A more detailed discussion of the changes with age for individual forms is given in Schaller (1972).

13. The forms which might be considered letter-like in varying degrees are Forms $1,14,19,35,37,38$, and 39, which resemble the letters I, V, D, i, T, C, and Y, respectively. In addition, Forms 26 , $25,24,23$, and 38 , in that order, might be considered to be increasingly similar to the letter "C."

(Received for publication July 19, 1974; accepted September 13, 1974.) 